\title{
Mobile Learning Systems in Software Engineering Education
}

\author{
Liliya Andreicheva \\ Institute of Computer Science \& Information Technologies \\ Kazan Federal University \\ Kazan, Russia \\ liliya.andreicheva@gmail.com
}

\author{
Rustam Latypov \\ Institute of Computer Science \& Information Technologies \\ Kazan Federal University \\ Kazan, Russia \\ roustam.latypov@kpfu.ru
}

\begin{abstract}
The latest achievements in the information sciences area are mostly connected with the mobile technologies. The significant growth in this area attracts more and more users worldwide. In this paper we concentrate on the mobile learning aspect in education process, specifically in learning management systems. We propose a new design approach which consolidates different front-end representations of the learning system with the single back-end instance. To increase the efficiency of the system we consider new module like preliminary homework checking module, which will be useful especially in software engineering field, and additional statistical and feedback modules.
\end{abstract}

Keywords-m-leaning; e-learning; mobile technologies; LMS; CMS; learning system; education; software engineering.

\section{INTRODUCTION}

In recent years there has been a tremendous growth of mobile technologies worldwide. Nowadays every day the technologies are getting more and more advanced. Modern people use smartphones and tablets almost everywhere - at work, at home, at university, etc. They read newspapers and journals online, check their e-mails while driving back home from work, post in social networks, and play games and chat. According to statistical information [1] the influence of the mobile devices will keep increasing in the nearest future. As we observe most of the users of popular gadgets are young people, students or pupils. This gives us an idea that we can turn mobile technologies into a powerful tool in the educational process.

Obviously, education is one of the most important steps in each young person's life. Unfortunately, not everyone understands it and as the result more effort is needed from the teachers and professors. As mobile technologies cause a lot of interest in the modern society, this fact can be used as an advantage in the educational process. We propose new design principles for the educational systems, whose main idea lies in the unity of back-end and variety of front-end forms. The common server part and the different ways to access information from the client side increases the educational system availability with the minimum set of requirements (basically you need just an Internet access from your mobile device). This paper consists of three parts. In the first part we define the main principles of the e-learning system usage, especially in the software engineering field. Second part contains some analysis of usage of electronic learning systems in Russia and worldwide. The third part is dedicated to our proposal of new design principles.

\section{E-LEARNING AND M-LEARNING}

Soon after an expansion of the computer technologies, the e-learning terminology became regular in educational process. Although in recent years as a product of the growth of the mobile industry we received a new term - m-learning. What is m-learning? M-learning states for mobile learning, which suggests the learning process is organized with the usage of mobile devices. This fact makes it available for everyone, who has a smartphone or a tablet. One of the greatest advantages of $\mathrm{m}$-learning is that it is independent from time and place.

The next question is why we need anything else if mlearning systems are available at any time and any place? Each method has its own advantages and disadvantages. The approach is based on the principles of work of different devices. For example, it is much more convenient to read a book from tablet than from smartphone. Although if you want to download this book and use information from it in you report, like different citations and images, you need a good text editor, which is most likely installed on your PC. The main idea is the consolidation of all forms of front-end to obtain the most efficient system for students and professors. These technical specifications define a set of requirements to the system, which we will discuss in the third part of this paper.

One of the drawbacks of the e-learning process is that it is not equally efficient in different areas of study. For example, medical disciplines which require a lot of practical exercises and direct communication with patients cannot be fully automated within the e-learning process. Let us look specifically on the software engineering (SE) area. The area itself is very wide and contains a lot of subjects that should be given to the student. An important aspect that is giving SE a great advantage in the automation of learning systems is that most of the subjects are technical: mathematics, physics, programming sciences, etc. The distinctive characteristics and specifics of this type of sciences allow simplifying the control units significantly. Various tests, quizes, multiple-choice questions with strict answers can be created easily for any 
software engineering course, while for art course you should show your personal vision of the topic and there is generally more than one correct answer. Therefore we propagate a wide usage of e-learning and m-learning technologies in the SE area.

Speaking in terms of international practices, we see that the major universities in the world support a lot of various programs based on the e-learning methods. Statistical analysis shows that every year more and more students enroll in online university services provided by the best universities in the world. This is a great opportunity for students with disabilities, international students and those who cannot afford to pay full tuition for getting the degree on campus. Unfortunately, nowadays leading universities in Russia are just starting to deploy the e-learning systems. Speaking about m-learning, for Russian universities it is still a future project. In my opinion we should start working actively in this area, because now this is a perspective future. We can see the growth of usage of the mobile application that has been deployed on campuses of American universities and colleges in 2010 and 2011 (see "Fig, 1") [2]. Based on the graph data, we can observe that values have doubled within a single year. Taking into consideration the speed of the development of the mobile technologies, we need to act fast to be able to compete with top universities in the world.

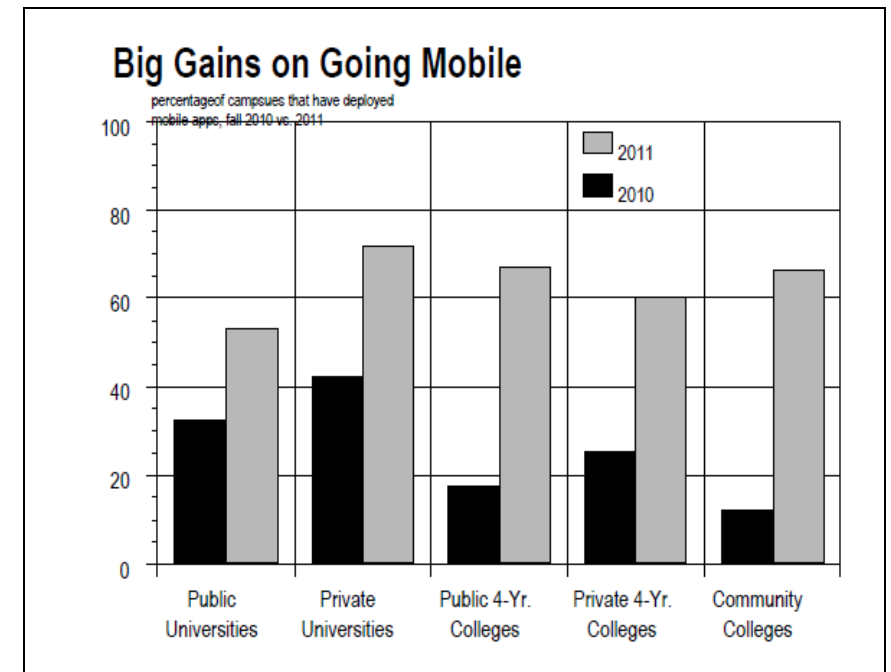

Fig. 1. Big Gains on Going Mobile, percentage of campuses that have deployed mobile apps, fall 2010 vs fall 2011 [2].

\section{Usage of Mobile Technologies}

According to statistics [3], US, Japan and South Korea are top most consumers of the mobile markets. The forecasted revenue is about $\$ 14$ billion by 2014 . This number explicitly shows the size of the market and its growth. Although the research results say that more than $22 \%$ of the Internet users in cities with population over 100,000 people in Russia access the Web from mobile devices, e-learning and m-learning technologies are not so widespread.

In recent years education became one of the prior areas in the country. In Soviet Union the level of education was one of the highest in the world. The hard political times on the edge of the centuries destroyed the educational system. Nowadays the best universities in the country should work a lot to achieve the same level that foreign schools are offering. Thus the area of elearning has become very important. If we look at the way the process is organized abroad we can see that in most of universities there is an internal system for students and professors, which offer a variety of options, from enrollment and scheduling to taking courses online and making presentations online. This is a very important step to every school. In general e-learning process attracts a lot of new students to universities, increases its popularity worldwide and makes education more approachable and affordable for different groups of people. The initial integration of new technologies will definitely require some investments but all the costs will be reimbursed later. For students taking online courses also has some financial benefits. There is a good example the savings the famous company IBM achieved using e-learning practice. In their training process for new managers they used online learning approach and it saved them more than 24 million US dollars. The new technologies used allowed to give 5 times more information and at the same time the cost for one study day was reduced from $\$ 400$ to $\$ 135$. The other advantage of e-learning approach is the variety of forms that can be integrated in the educational process. The simplest one will be usage of course management and learning management systems (CMS and LMS) in the specific course. More solid approach is the application for the whole set of subjects, which prepare a student for some degree or certification exam. In Russia this is a rare practice, but in our opinion this is the future of the educational process for big universities.

Let us specify some use cases for the e-learning system to obtain the set of requirements. We consider two main roles in the system: professor and student. There are a lot of different aspects that should be taken into consideration in the system, but in the use cases we concentrate on the aspects that are important in the m-learning scope.

One of the regular situations is that professor has to make some changes in the schedule and this information should be sent quickly. Mobile technologies solve this question easily. Various methods can be implemented, via e-mail, via text messages in the message exchange system, but as everybody now has a mobile phone next to him/her all the time, the push messages from the mobile application are the easiest solution. The mechanism of push messages can be used in many cases; therefore there should be a configurable notification system, which will allow providing the most recent information to both professors and students as soon as any change occurred. As we have mentioned before schedule update events are very important, information that new learning materials are available or homework grades are posted. Students are usually mostly interested in their grades and they want to know this information as soon as possible. So it is more convenient for them to check the mobile application rather than to wait till they get back home and log in to the e-learning system on their PC. The specific use of notifications for professors can help to track if all the homework was submitted on time. Another example will be notifications from the administrator of the system about technical issues, like "Servers A and B will be rebooted in 5 minutes. Do not run any tasks at that time", etc. The advantage of using mobile technologies here is the independence from place and time of the target object of 
notification and the speed. Generally viewing message from the mobile app is faster than checking an e-mail or viewing message through the LMS.

Another important aspect, especially for students, is the availability of the materials. Imagine a student, who is working for some company and is getting a degree at the same time. In Russia this is a very widespread situation. The student spends a lot of time in transport, it can be either public transportation, which is usually slow, or traffic jams.

But he does not want to lose this time, he better review once again lectures for the test, which is later the same day. In this case using laptops is not a suitable solution as they are still big and heavy enough. Tablets and smartphones can easily solve the problem, even when you are driving you can enable the voice support on the device, so that it will be reading text to you aloud. By the way, here we should also take into consideration the technical specifications of different gadgets. It is not always convenient to read from you smartphone if the screen is not big enough, but if you have the headset, you can listen to the same lecture. Or you can watch the video recorded during the lecture. Tablets are more user-friendly for these specific purposes. For professors access to homework assignments and tests is more valuable. Thus when they get into the same situation with transportation they can look through the homework tasks or run some automated procedures that check tests and quizzes submitted by the class. These actions can be performed by one button click, they start the job on server and by the time professor gets home he already has a report with the results of the last test. This kind of automation saves a lot of time, which allows professors to spend it later on research and student questions.

These examples show the particular use cases for $\mathrm{m}$ learning, but we have other forms of the front-end, like webbased console and regular PC program. In the next part we will discuss how the whole system should be working together.

\section{System Design}

The main idea of our proposal is to have the unified concept for different representations of the front-end of the application. This will allow organizing the LMS and CMS in the rational and user-oriented way. The general practice shows that it is much more difficult to upgrade the existing software, which may be absolutely unsuitable for the new needs. Thus a good design approach should specify all the use cases and the corresponding requirements in advance. This is one of the reasons why the idea of development of new system is better than using some existing ones. An individual project can be turned up for the specific goals, while any modification of an existing tool presented on the market is a long time- and laborconsuming process. The other reason is that most of the existing systems are expensive. Although there is an opensource segment of the market, if we look at the theory of the delayed expenses this approach becomes inapplicable for large educational organizations like universities and colleges. At some point there is always a risk that developers of opensource software will decide to stop the support of this particular product that you are using. This can cause a lot of problems for the users, as eventually it leads not only to the change of an existing system but also to the migration of the information from one system to another, which is also expensive and inconvenient.

Although we consider three types of representation of the user interface for the system (see "Fig. 2"), we concentrate mainly on the mobile application and PC programs. The reason for our choice is that these two parts have different purposes. As we have mentioned earlier the use cases for the m-learning application first of all allow the user to be independent from time and location. The set of features for this app will be defined mostly by this factor, however we also take into account that some actions are inconvenient to perform from the mobile device (like file upload, download, text/graph editing, submission of programming assignments, etc.). For the PC we consider a complete system with support of a variety of features for students, professors and administrators. As we have mentioned several roles above, let us elaborate on that.

We have already mentioned students and professors as the target users of the application. Besides them there is one more user role - administrator, who is responsible for registration of other users, helping them with any questions about system usage and controlling the whole system. In general we suppose that system includes plenty of courses from various departments. To handle the department information accurately and to be able to control the department schedules we propose a subgroup of administrators called department administrators. Basically the administrator can create another administratoruser who will be able to work only with data from his department (see "Fig. 3"). One of the possible scenarios of system development is integration with some other useful services of university campus, for example, information about operation hours of departments, library, on-campus cafes, etc. In this case the role of an administrator will be to include handling information for these additional services. We will not discuss the administrator role a lot because he is basically only using the PC application, while in this paper we concentrate on the m-learning aspect.

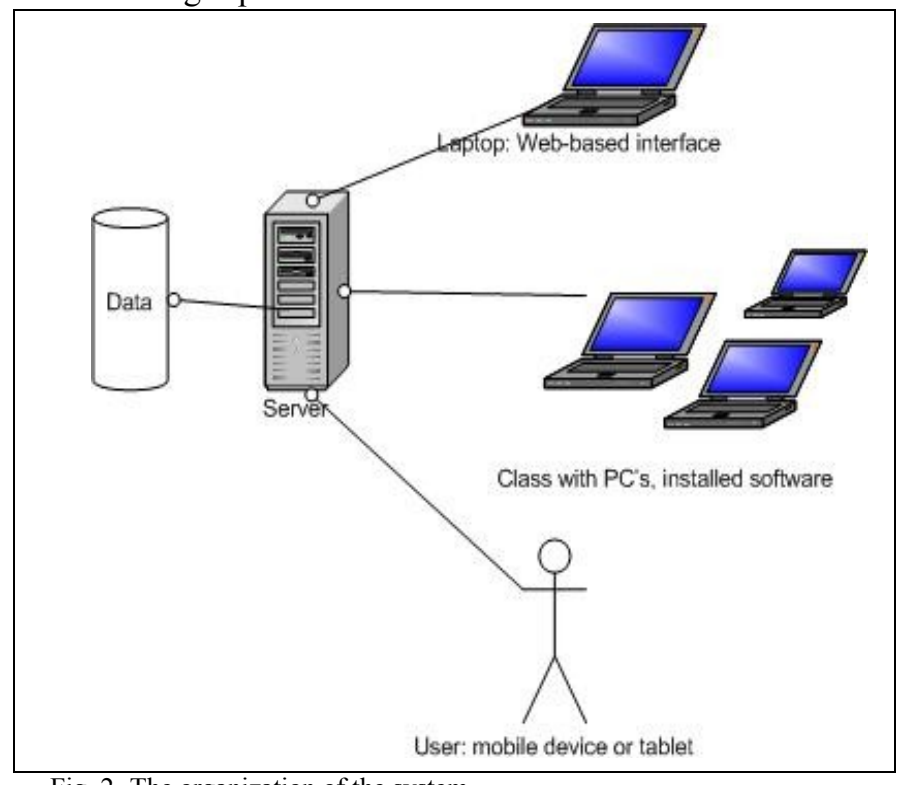

Fig. 2. The organization of the system. 


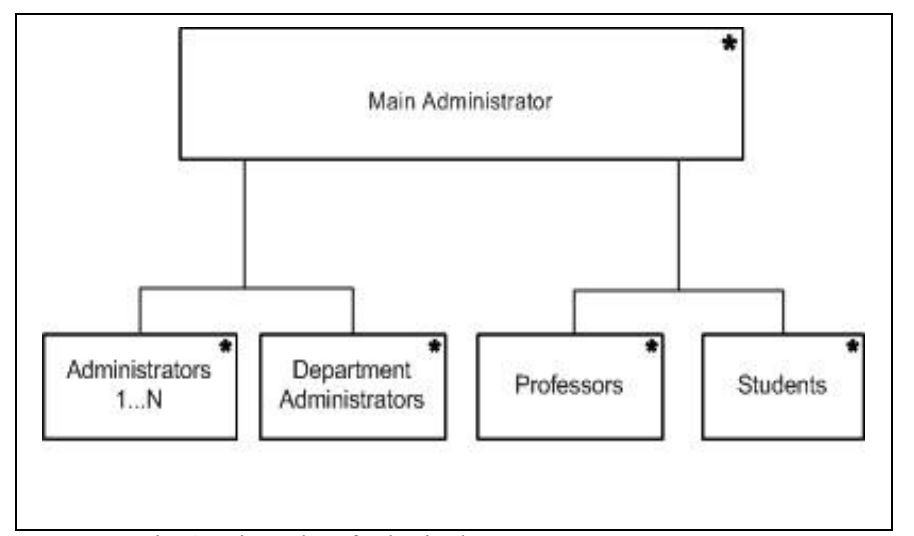

Fig. 3. Hierarchy of roles in the system.

Based on the use cases that we have mentioned above the primary purpose of the mobile application is to keep the user updated with the latest progress that is going on in the system. Thus we should concentrate on the notification system and monitoring console. From our point of view notification mechanism is more important for students, while monitoring is more valuable for professors. Nevertheless in the design we consider both types of users being able to use these components.

Generally notifications can be divided into two types: created by system and created by user. Each of these types of messages includes the following levels: information, warning, error. For better user experience we also suggest three types of importance of every message (low, regular, high). This makes system more flexible, because depending on the user's approach he can configure the individual preferences. The importance level will help the use handle urgent questions with high priority faster. On the other hand, from the system development point of view, such notification mechanism will help improve the product stability and troubleshooting. The system notifications are configured by administrator. They are designed mainly for administrators to keep the system maintained. But regular users should also be aware of the current situation. For example, when student is trying to upload his homework but the server is rebooting, he should get an appropriate alert to be able to perform this operation later. This will be a user-friendly behavior and will decrease the number of errors. The user should be able to set up notification mechanism in general and for any particular course. The most important issues from the system point of view include system failures, database failures, server connection problems, protocol problems, running jobs problems (running automated homework or quiz checking, etc.), user access problems, etc. The alert message should contain description of the problem, error code if applicable, and suggestions of possible solution. The user defined notifications include information about any changes in the course schedule, homework assignments, lecture materials, forum updates, tests and examination results, course announcements, etc. The set of these messages should be the same for students and professors. As it might be the case where one course is divided between two professors and both have access to course materials, so one should be notified of what another is changing in the course.
Monitoring is a great tool mostly for professors. Besides some general statistical information about popularity of the course, individual student performance, overall student performance, etc., provided logical set of rules will be able to analyze performance of students in general and individually within the course and give some recommendations. For example, module A was successfully learned by $90 \%$ of students, $5 \%$ need to review the module once again, 5\% finished the module with average knowledge of the subject. This information should be interesting to both teachers and students. From the development point of view it will require implementation of some analytical algorithms on the back-end. More complex approach can involve some expert systems or self-learning neural networks. Monitoring of individual performance is also very important for students. We should remember that one of the main characteristics of the e-learning process is individual factor. The whole concept of e-learning supposes that most of work is done individually and professor is controlling it remotely. This requires good selforganizational abilities, time management and motivation. Thus the overall performance of the student should be given in comparison to the class that will increase student's motivation.

One of the most important components of educational process is control of the level of knowledge obtained, thus we consider in e-learning systems particular attention should be paid to this aspect. Information technologies brought automation to the regular slow processes and this greatly increased the performance in various areas. Therefore, the higher is the level of automation of the system, the easier and more practical it is in use. Our idea supposes adding some extra control on the stage of homework submission. We offer a module which will execute some preliminary control. The goal of this module is to have some self-controlling tool for the students. The main idea of this approach is that the system performs some additional checks when uploading homework. The result is an informational message demonstrating the validity of the submitted task. Based on the response of the system student can then re-upload his homework with any modifications needed, if the original exercises have been carried out not quite right. To implement this approach, a set of tests to verify the job is needed. Such set can have quite different views. From basic check for the correct answers of the math problems to various tests for the correctness of the output of some programs delivered as assignments for programming courses. This module will depend a lot on the particular subject and the approach of the professor. This tool will be easy to configure for the various software engineering courses. Overall the effort for making this module work properly in technical courses is less, than it would be for art courses. Nevertheless we consider this approach useful in every area. Basically the efficiency of the module depends on the professor. For programming courses, for example, the control condition to the minimum practical tasks may be simply an ability to compile the program, and more serious approach will have some test units, checking the correctness of the input parameters, output results and test the operation of the program on different operating systems, etc. From the students point of view this module is very practical and provides additional help. Obviously with proper organization of the time they can upload the homework in advance to the system and 
get a preliminary result. Now they know whether their work fits the minimum requirement for this task. Such verification system does not involve full check of the assignment on-a-fly, but allows students to significantly improve their results. For professors module also provides an effective time management: time taken to prepare the preliminary tests for jobs will reduce the time needed to check homework, and improve the quality of the provided solutions.

Nowadays one of the common methods of improving something is polling the audience. Thus in many areas system of feedback and suggestions is used. From our point of view, the presence of such a system will make the e-learning system more effective. The use of such a module in e-learning systems is simple for users, thanks to the process automation; the results are calculated quickly and immediately available for the professor. This module performs several functions:

- Evaluation of the professor - how effective his method of teaching is, whether he explains the material good enough, whether he answers the questions and emails quickly, etc.

- Course evaluation - whether course content suites expectations, do selected materials cover the subject, were the home assignments effective, how is the control system, how popular is this course among students, etc.

The big advantage of this survey is that it may be configured from the same set of questions regardless of the course topics, and include some specific items that will be important for a particular teacher and course. If there is no such a need for the specific questions, the section where comments allowed can help a lot. The generic system of evaluation of courses and instructors can be organized when using same questions in the surveys. This approach allows creating a rating system. Rating will help students to select from a variety of courses, instructors. For the organizations, that use e-learning system for professional trainings rating system will help to evaluate the effectiveness of professors and courses.

From the developer's point of view an e-learning system is a complex application primarily divided into front-end and back-end. In our case we are basically talking about a clientserver architecture where the client side has different views. On the one hand, the client is a separate application for the PC, on the other hand, it is a mobile application, which uses a completely different design principles. However, both of them are parts of the same system, working with a server, which stores all the data. As we have mentioned earlier we concentrate primarily on the mobile and PC client views, but as another view we consider a web console. Running an elearning system from the browser also requires some other technologies. At this point an important issue is to keep the client-server protocol the same for communication between all parts of the application. One of the other important questions is synchronization. When using file-sharing service Dropbox, files are downloaded from one device to the shared folder, and then are displayed at all devices that run with the appropriate application. The same idea should be used in the proposed system. So before actual implementation is done, a careful study of the mathematical model technologies should be done, because every part of the application is very different.

In this paper we present the system design. The initial prototype is under construction now. However, the described approach presents fully the main features of the whole system. The heart of the system is the server part, which takes most of the development time. Overall, development process contains a lot of pitfalls, which are connected with different problems from the technologies point of view and

The novelty of the solution lies not only in the choice of a new long-term approach to client-server architecture, but also in the introduction of the new modules for the convenience of teachers and students. Our design approach is promising in terms of end-user response, as it is a priority to increase the availability and convenience of e-learning systems for users. Based on statistical data [1], we conclude that the audience of Internet users and advanced mobile devices is increasing. Education has always been a key element in the development of the society. Thus, the introduction of the latest technology innovations in the educational process can only increase the development of the state and society.

\section{REFERENCES}

[1] Blinov, D. (2012). Statistics of usage of mobile devices, platforms and applications. Retrieved

from: http://beamteam.ru/2012/09/mobile-platforms-share-2012/

[2] A Profile of the LMS Market (page 18), CampusComputing, 2011. Retrieved from: http://www.campuscomputing. net/sites/www.campuscomputing.net/files/Green-

CampusComputing2011_4.pdf

[3] Nicole Fougere, US Leads the Global Mobile Learning Market, 2010 http://www.litmos.com/mobile-learning/us-leads-the-global-mobilelearning-market-mlearning/

[4] Kerschenbaum, Steven (04). "LMS Selection Best Practices" (White paper). Adayana Chief Technology Officer. pp. 1-15, 13 February 2013. Retrieved from: http://www.trainingindustry.com/media/2068137//msselection_full.pdf

[5] Ellis, Ryann K. (2009), Field Guide to Learning Management Systems, ASTD Learning Circuits. Retrieved from: http://www.astd.org/ /media/Files/Publications/LMS fieldguide_20091

[6] Wikipedia. http://www.wikipedia.org/ 